\title{
Genetic modifiers of CHEK2*1100delC-associated breast cancer risk
}

Taru A. Muranen, MSc'1, Dario Greco, $\mathrm{PhD}^{2}$, Carl Blomqvist, MD, PhD³, Kristiina Aittomäki, MD, $\mathrm{PhD}^{4}$, Sofia Khan, PhD ${ }^{1}$, Frans Hogervorst, $\mathrm{PhD}^{5}$, Senno Verhoef, MD ${ }^{5}$, Paul D.P. Pharoah, MB, BCh ${ }^{6,7}$, Alison M. Dunning, $\mathrm{PhD}^{6}$, Mitul Shah, $\mathrm{MSc}^{6}$, Robert Luben, $\mathrm{BS}^{8}$, Stig E. Bojesen, MD, PhD 9,10,11, Børge G. Nordestgaard, MD, DMSc 9,10,11, Minouk Schoemaker, PhD ${ }^{12}$, Anthony Swerdlow, DM, DSc ${ }^{12,13}$, Montserrat García-Closas, PhD ${ }^{12,14}$, Jonine Figueroa, PhD $^{14}$,

Thilo Dörk, PhD ${ }^{15}$, Natalia V. Bogdanova, PhD ${ }^{16}$, Per Hall, MD ${ }^{17}$, Jingmei Li, PhD ${ }^{17}$, Elza Khusnutdinova, MD ${ }^{18,19}$, Marina Bermisheva, PhD ${ }^{15,19}$, Vessela Kristensen, PhD $^{20-22}$, Anne-Lise Borresen-Dale, PhD ${ }^{20,22}$, NBCS Investigators ${ }^{20-34}$, Julian Peto, PhD $^{35}$, Isabel dos Santos Silva, PhD ${ }^{35}$, Fergus J. Couch, $\mathrm{PhD}^{36}$, Janet E. Olson, PhD ${ }^{37}$, Peter Hillemans, $\mathrm{PhD}^{15}$, Tjoung-Won Park-Simon, MD ${ }^{15}$, Hiltrud Brauch, PhD ${ }^{38-40}$, Ute Hamann, PhD ${ }^{41}$,

Barbara Burwinkel, PhD ${ }^{42,43}$, Frederik Marme, MD ${ }^{43,44}$, Alfons Meindl, PhD ${ }^{45}$, Rita K. Schmutzler, MD ${ }^{46-48,}$ Angela Cox, $\mathrm{PhD}^{49}$, Simon S. Cross, MD ${ }^{50}$, Elinor J. Sawyer, $\mathrm{PhD}^{51}$, lan Tomlinson, $\mathrm{PhD}^{52}$, Diether Lambrechts, PhD ${ }^{53,54}$, Matthieu Moisse, PhD ${ }^{53}$, Annika Lindblom, MD ${ }^{55}$, Sara Margolin, MD ${ }^{56}$, Antoinette Hollestelle, PhD ${ }^{57}$, John W.M. Martens, PhD ${ }^{57}$, Peter A. Fasching, MD ${ }^{58,59}$, Matthias W. Beckmann, MD ${ }^{58}$, Irene L. Andrulis, PhD ${ }^{60,61}$, Julia A. Knight, PhD ${ }^{62,63}$, kConFab/AOCS Investigators ${ }^{80}$, Hoda Anton-Culver, $\mathrm{PhD}^{64}$, Argyrios Ziogas, $\mathrm{PhD}^{64}$, Graham G. Giles, PhD ${ }^{65,66}$, Roger L. Milne, PhD ${ }^{65,66}$, Hermann Brenner, MD, MPH $38,67,68$, Volker Arndt, MD, MPH ${ }^{68}$, Arto Mannermaa, PhD ${ }^{69-71}$, Veli-Matti Kosma, MD ${ }^{69-71}$, Jenny Chang-Claude, PhD ${ }^{72}$, Anja Rudolph, $\mathrm{PhD}^{72}$, Peter Devilee, PhD ${ }^{73,74}$, Caroline Seynaeve, MD, PhD ${ }^{57}$, John L. Hopper, PhD ${ }^{65}$, Melissa C. Southey, PhD ${ }^{75}$, Esther M. John, PhD ${ }^{76,77,78,}$ Alice S. Whittemore, PhD77,78, Manjeet K. Bolla, MSc7, Qin Wang, MSc7, Kyriaki Michailidou, PhD ${ }^{7,79}$, Joe Dennis, $\mathrm{MSc}^{7}$, Douglas F. Easton, PhD ${ }^{6,7}$, Marjanka K. Schmidt, PhD ${ }^{5}$ and Heli Nevanlinna, PhD ${ }^{1}$; on behalf of the Breast Cancer Association Consortium

Purpose: $C H E K 2^{\star} 1100$ delC is a founder variant in European populations that confers a two- to threefold increased risk of breast cancer (BC). Epidemiologic and family studies have suggested that the risk associated with $C H E K 2^{\star} 1100$ delC is modified by other genetic factors in a multiplicative fashion. We have investigated this empirically using data from the Breast Cancer Association Consortium (BCAC).

Methods: Using genotype data from 39,139 (624 1100delC carriers) BC patients and 40,063 (224) healthy controls from 32 BCAC studies, we analyzed the combined risk effects of $C H E K 2^{\star} 1100$ delC and 77 common variants in terms of a polygenic risk score (PRS) and pairwise interaction.

Results: The PRS conferred odds ratios (OR) of 1.59 (95\% CI: $1.21-$ 2.09) per standard deviation for BC for $C H E K 2^{\star} 1100$ delC carriers and $1.58(1.55-1.62)$ for noncarriers. No evidence of deviation from the multiplicative model was found. The OR for the highest quintile of the PRS was 2.03 (0.86-4.78) for CHEK2*1100delC carriers, placing them in the high risk category according to UK NICE guidelines. The OR for the lowest quintile was 0.52 (0.16-1.74), indicating a lifetime risk close to the population average.

Conclusion: Our results confirm the multiplicative nature of risk effects conferred by $C H E K 2^{\star} 1100 \mathrm{delC}$ and the common susceptibility variants. Furthermore, the PRS could identify carriers at a high lifetime risk for clinical actions.

Genet Med advance online publication 6 October 2016

Key Words: breast cancer; Breast Cancer Association Consortium; CHEK2 ${ }^{\star} 1100$ delC; common variants; polygenic risk score 
The protein-truncating mutation $C H E K 2^{\star} 1100$ delC (checkpoint kinase 2) is a moderate-penetrance breast cancer risk variant with an estimated relative risk of two- to threefold., ${ }^{1,2}$ However, several studies have shown that the cumulative lifetime risk of breast cancer for $C H E K 2^{*} 1100$ delC carriers is markedly higher in women with a family history than in those without, ${ }^{3-5}$ and that $C H E K 2^{\star} 1100$ delC carriers have a higher probability of developing bilateral breast cancer. ${ }^{6}$ These observations are quantitatively consistent with a simple polygenic model suggesting that $\mathrm{CHEK}^{\star} 1100 \mathrm{delC}$ combines multiplicatively with other genetic loci. However, this has not yet been established empirically.

Genome-wide association studies have identified common genetic variants that are associated with increased risk of breast cancer. A polygenic risk score (PRS) based on 77 lowpenetrance variants may explain approximately $12-14 \%$ of the excess familial risk and has been shown to identify individuals at high risk at the population level. ${ }^{78}$ Some of these variants predispose predominantly to either estrogen receptor-positive $(\mathrm{ER}+)$ or estrogen receptor-negative (ER-) disease, the two main etiological subclasses of breast cancer. ${ }^{9}$ CHEK $2^{\star} 1100 \mathrm{delC}$ carriers are more strongly predisposed to $\mathrm{ER}+$ disease: approximately $90 \%$ of carrier tumors are $\mathrm{ER}+$ in comparison to $77-78 \%$ of noncarrier tumors. ${ }^{10}$

Here, we report the synergistic risk effects attributable to CHEK2 $2^{\star} 1100$ delC and the common breast cancer susceptibility variants individually and summarized in terms of the PRS. ${ }^{7,8}$

\section{Study participants}

MATERIALS AND METHODS

Female patients with invasive breast cancer and healthy controls of European ancestry from studies participating in the Breast Cancer Association Consortium (BCAC) were included (Supplementary Table S1 online). Data from a study were included if the study provided genotype data of the common variants from at least one breast cancer patient carrying the $1100 \mathrm{delC}$ variant. This selection yielded data from 32 studies and a total of 79,202 study subjects, including 848 CHEK2 ${ }^{\star} 1100$ delC carriers (Supplementary Table S2 online) for pairwise interaction analyses. Complete quality-controlled ${ }^{7,10}$ genotype data for all common variants and $C H E K 2^{\star} 1100$ delC were available from 33,624 study subjects (369 CHEK2*1100delC carriers, Supplementary Table $\mathbf{S 2}$ online). These data were used in the analyses involving the PRS.

All participating studies were approved by their institutional review committees. Each study followed national guidelines for participant inclusion and informed-consent procedures.

\section{Genotyping}

All variants except $C H E K 2^{\star} 1100$ delC were genotyped centrally using a custom Illumina iSelect genotyping array (iCOGS, Illumina, San Diego, CA) as part of the COGS consortium studies as described. ${ }^{7.8}$ CHEK2 $2^{\star} 1100 \mathrm{delC}$ was genotyped primarily using a custom-made TaqMan assay (Applied
Biosystems, Foster City, CA); a small minority was genotyped using iPLEX. ${ }^{10}$ In addition to the 38,549 study subjects genotyped using the iCOGS array, 40,653 BCAC study subjects were genotyped for up to 25 of the common risk variants. These data were used in the pairwise interaction analysis (Supplementary Tables S2 and S3 online). These samples were genotyped by independent studies following BCAC genotyping standards as described previously. ${ }^{11,12}$

\section{Statistical analyses}

Statistical analyses were performed using Stata SE 10 (StataCorp, College Station, TX) and R version 2.15.2 (R Foundation for Statistical Computing, Vienna, Austria). For the common variants, a log-additive model was assumed; i.e., the risk was analyzed in terms of the number of disease-associated alleles $(0,1$, 2) carried. CHEK2 $2^{\star} 1100 \mathrm{delC}$ was assumed to follow a dominant inheritance model because the number of rare homozygotes was small $(n=19)$. All analyses adjusted for the study and seven principal components defined on the basis of the genome-wide data from the iCOGS project as described previously. ${ }^{7}$ All reported tests were two-sided.

\section{Polygenic risk score}

To investigate the combined effects of common variants and CHEK2*1100delC, a polygenic risk score (PRS) based on the main effects of the common variants was calculated using the formula $\sum_{i=1}^{n} a_{i} \log _{2} O R_{i}$, where $n$ is the number of loci included in the model, $a$ is the number of susceptibility alleles in locus $i$, and OR is the per-allele odds ratio for breast cancer, estimated separately for each variant in the entire data set (Supplementary Table S4a online, "All" column). Results using a PRS based on previously reported $\mathrm{ORs}^{7,8}$ were essentially identical (data not shown). The PRS was approximately normally distributed in all study subgroups and it was standardized by the mean and standard deviation of the PRS among healthy individuals. ${ }^{8}$ For pairs of linked variants with $\mathrm{r}^{2}>0.75$, we included in the PRS only the lead variant (rs2981579, not rs2981582; rs12662670, not rs3757318; rs554219, not rs614367). We excluded two variants (rs78540526 and rs75915166) included in the PRS used by Mavaddat et al. ${ }^{8}$ (which were not genotyped on the iCOGS array) as well as rs17879961, the CHEK2 missense variant I157T, because the number of study subjects carrying both $1100 \mathrm{delC}$ and I157T was very low $(n=5)$. Thus, the resulting PRS included 74 variants. The interaction between PRS and CHEK2 $2^{\star} 1100$ delC was assessed by comparing the following nested logistic regression models: a model including the PRS and 1100delC genotype and a model supplemented with an interaction term coded as the product of the PRS and 1100delC. In the analyses of the PRS and positive family history of breast cancer, positive family history was defined as at least one firstdegree relative with breast cancer.

The cumulativelifetime breast cancer risk of $\mathrm{CHEK} 2^{\star} 1100 \mathrm{delC}$ carriers in different PRS percentiles was derived by assuming 
an average lifetime risk of $22 \%$ for $\mathrm{CHEK} 2^{\star} 1100 \mathrm{delC}$ carriers ${ }^{13}$ and previously published relative risk estimates associated with the PRS. ${ }^{8}$

\section{Pairwise interaction analyses}

We tested for pairwise interaction between each common variant and $C H E K 2^{\star} 1100 \mathrm{delC}$ as described for the interaction between the PRS and 1100delC. $P$ values were corrected for 77 parallel tests using the Benjamini-Hochberg method. ${ }^{14}$ The OR for breast cancer was estimated separately for each of the common variants for the whole dataset and for the subgroup of $1100 \mathrm{delC}$ carriers. These analyses were also performed separately using a subgroup of breast cancer patients with ER+ disease because $1100 \mathrm{delC}$ is associated with ER+ breast cancer. ${ }^{10}$ We tested for heterogeneity in the ORs among different BCAC studies by including (separately for each variant) an interaction term between the variant and the study. No significant heterogeneity was found for any variant (data not shown). Statistical power was estimated as previously suggested for risk interaction analyses. ${ }^{15}$

\section{RESULTS}

We analyzed the combined effects of CHEK2*1100delC and common low-penetrance breast cancer risk variants using data from the international Breast Cancer Association Consortium (Supplementary Table S2 online). The PRS summarizing the individual effects of 74 common variants was strongly associated with breast cancer risk among $C H E K 2^{\star} 1100$ delC carriers (OR per unit of standard deviation $1.59(1.21-2.09), P=0.0008$ ) and the OR was similar to that of noncarriers (1.58 (1.55-1.62), $\left.P_{\text {interaction }}=0.93\right)$. ORs for the highest and lowest quintiles of the PRS distribution were $2.03(0.86-4.78)$ and $0.52(0.16-1.74)$ for CHEK $2^{\star} 1100$ delC carriers, respectively, when compared to the middle quintile (Table 1). Both estimates were similar to those among noncarriers.

The OR associated with CHEK2* $1100 \mathrm{delC}$ in the analysis data set $(2.99(2.32-3.85))$ was attenuated when the model was adjusted for positive family history of breast cancer. The OR associated with the PRS was also slightly attenuated (Table 2).

Table 1 Breast cancer risk associated with the PRS for noncarriers and the carriers of CHEK2*1100delC

\begin{tabular}{|c|c|c|c|c|}
\hline & Noncarriers & & $\begin{array}{c}\text { CHEK } 2 * 1100 \text { delC } \\
\text { carriers }\end{array}$ & \\
\hline & OR $(95 \% \mathrm{Cl})$ & $P$ & OR $(95 \% \mathrm{Cl})$ & $P$ \\
\hline PRS $^{a}$ & $1.58(1.55-1.62)$ & $<1.0 \mathrm{E}-10$ & $1.59(1.21-2.09)^{b}$ & 0.0008 \\
\hline Percent & f PRS, \% & & & \\
\hline$<20$ & $0.52(0.48-0.56)$ & $<1.0 \mathrm{E}-10$ & $0.52(0.16-1.74)$ & 0.29 \\
\hline $20-40$ & $0.78(0.72-0.84)$ & $2 \mathrm{E}-11$ & $0.72(0.28-1.88)$ & 0.51 \\
\hline $40-60$ & Referent & & Referent & \\
\hline $60-80$ & $1.25(1.16-1.34)$ & $8 \mathrm{E}-10$ & $0.93(0.39-2.25)$ & 0.88 \\
\hline$>80$ & $1.92(1.80-2.06)$ & $<1.0 \mathrm{E}-10$ & $2.03(0.86-4.78)$ & 0.11 \\
\hline
\end{tabular}

OR, odds ratio; PRS, polygenic risk score.

${ }^{\mathrm{a}} \mathrm{OR}$ was estimated per unit of standard deviation of the PRS. ${ }^{\mathrm{b}} P$ value $=0.93$ for pairwise interaction between CHEK2*1100delC and PRS.
No significant interaction between risk effects associated with 1100delC, PRS, and positive family history was found. However, in a case-only analysis, there was a significant association between the PRS and family history of breast cancer among both CHEK2*1100delC carriers (OR 1.29 (1.01-1.65), $P=0.04)$ and noncarriers (OR 1.17 (1.12-1.21), $P=4 \mathrm{E}-16)$ (Supplementary Figure S1 online).

When 77 common variants were considered individually, we found nominally significant interactions between five variants and $C H E K 2^{\star} 1100$ delC for overall breast cancer (rs11249433, rs11780156, rs204247, rs2981582, and rs704010; Supplementary Table S4a online). Two of these represented synergistic (more than multiplicative) and three antagonistic interactions (the estimated effect in 1100delC carriers being in the direction opposite to that in noncarriers). However, none of the interactions was significant after correction for multiple testing. Nine variants showed a nominally significant interaction for ER-positive breast cancer (Supplementary Table S4b online).

\section{DISCUSSION}

Our analyses of the synergistic effects of CHEK2*1100delC and 77 common low-penetrance variants on breast cancer risk strongly support the predicted multiplicative polygenic model. ${ }^{8,16,17}$ Although this has previously been shown for combinations of low-penetrance variants ${ }^{8}$ and for variants in combination with BRCA1 and BRCA2 mutations, ${ }^{18}$ this is the first direct demonstration of a "moderate" risk gene; therefore, it has important implications for risk prediction. The PRS was a significant risk factor for $C H E K 2^{\star} 1100 \mathrm{delC}$ carriers, and the estimated OR per unit of standard deviation was very similar in $C H E K 2^{\star} 1100$ delC carriers and in noncarriers, consistent with the hypothesis that the common susceptibility variants combine with the rare $C H E K 2^{\star} 1100$ delC variant in an approximately multiplicative fashion. Similarly, the PRS risk estimates for the highest and lowest quintiles did not differ between the CHEK $2^{\star} 1100$ delC carriers and noncarriers. These two estimates for the $C H E K 2^{\star} 1100$ delC carriers alone did not reach statistical significance (Table 1), possibly reflecting limited statistical power due to the relatively low number of healthy variant carriers (Supplementary Table S2 online). However, this is the largest study genotyped for $C H E K 2^{\star} 1100 \mathrm{delC}$ and these

Table 2 Relative breast cancer risk associated with CHEK2*1100delC, PRS, and positive family history of breast cancer in the analysis data set

\begin{tabular}{lcccc} 
Risk model & Parameters & OR & $\mathbf{9 5 \%} \mathbf{C l}$ & $\boldsymbol{P}$ \\
\hline BC 1100delC + PRS & 1100delC & 2.99 & $2.32-3.85$ & $<1.0^{-10}$ \\
& PRS & 1.58 & $1.55-1.62$ & $<1.0^{-10}$ \\
BC 1100delC + & 1100 delC & 2.42 & $1.71-3.47$ & $9.4^{-7}$ \\
PRS + family history & PRS & 1.55 & $1.50-1.60$ & $<1.0^{-10}$ \\
& Family history $^{\text {a }}$ & 2.73 & $2.48-3.47$ & $<1.0^{-10}$ \\
\hline
\end{tabular}

OR, odds ratio; PRS, polygenic risk score.

aNo significant interaction between positive family history of breast cancer and either CHEK2*1100delC or PRS was found 
common variants. Even though some of the point estimates are not significant, they are consistent with previous reports. Most importantly, we did not find evidence for deviation from the multiplicative model, suggesting that the PRS could be used in risk stratification of 1100 delC carriers in a manner similar to that for noncarriers.

The unadjusted OR for the CHEK2*110delC variants (Table 2) was higher in our analysis data set than that in previous reports. ${ }^{2,13}$ Adjusting for positive family history markedly attenuated the CHEK2*1100delC-associated OR, suggestive of some oversampling of familial cases. The PRS OR was also slightly attenuated after the adjustment. However, CHEK2*1100delC, PRS, and family history remained significant risk factors in the combined model (Table 2), suggesting that the common variants together explain part of the excess familial risk as previously suggested, ${ }^{16}$ but that the PRS also has predictive value in breast cancer families segregating CHEK2 $2^{\star} 1100 \mathrm{delC}$.

Recently, a large study estimating the risk associated with CHEK $2^{\star} 1100$ delC in relation to age, tumor subtype, and family history reported that the cumulative lifetime risk for 1100delC carriers was approximately $22 \%{ }^{13}$ Assuming that the relative effect of the PRS is the same in carriers and noncarriers (OR $>1.48(1.39-1.57)$ or $<0.65(0.60-0.70)$ for percentiles $>80 \%$ or $<20 \%$, respectively), ${ }^{8} 20 \%$ of the 1100 delC carriers with the highest PRS would have a lifetime risk higher than $32.6 \%$ (30.6$34.5 \%)$, thus exceeding the threshold for the high-risk category (>30\%) according to the UK NICE guidelines for familial breast cancer. ${ }^{19}$ Similarly, for the $20 \%$ of 1100 delC carriers with the lowest PRS, the lifetime risk would be less than 14.3\% (13.2\%$15.4 \%)$, i.e., close to the average population risk. These observations imply that if $C H E K 2^{\star} 1100 \mathrm{delC}$ is to be used for risk prediction, then it can be made more effective by including the PRS, which represents the risk-modifying effects of common variants, in the prediction.

CHEK2 ${ }^{\star} 1100$ delC carrier cancers do not represent a phenotypically distinct subgroup of breast carcinomas. Instead, the phenotypic diversity of $C H E K 2^{\star} 1100$ delC-associated cancers resembles that of breast tumors in general. ${ }^{10}$ Thus, it was not surprising that the relative risks conferred by the common variants were similar for the $C H E K 2^{\star} 1100$ delC carriers and noncarriers, and that no significant pairwise interaction was found. We estimated that we had sufficient statistical power $(80 \%$, at $P<0.05)$ to detect a pairwise interaction between CHEK2 $2^{\star} 1100 \mathrm{delC}$ and any of the common variants if the interaction OR was 2.5 or more, but not enough power to detect interactions comparable in magnitude to the risk effects associated with the low-penetrance variants (OR 1.1-1.5). Thus, it remains possible that more modest departures from a multiplicative model may exist. However, if so, then much larger casecontrol studies, perhaps combined with pedigree analyses, will be required to detect them.

In conclusion, our analyses confirm the predicted multiplicative relationship between $C H E K 2^{\star} 1100 \mathrm{delC}$ and the common low-penetrance variants. Hence, the PRS could be applied to risk prediction of the variant carriers in a manner similar to that used for the general population. Most importantly, the PRS could help identify the high-risk group of the CHEK2*1100delC carriers who would benefit most from clinical intervention.

\section{SUPPLEMENTARY MATERIAL}

Supplementary material is linked to the online version of the paper at http://www.nature.com/gim

\section{ACKNOWLEDGMENTS}

Acknowledgments are linked to the online version of the paper at http://www.nature.com/gim.

\section{DISCLOSURE}

The authors declare no conflict of interest.

The last two authors contributed equally to this work.

${ }^{1}$ Department of Obstetrics and Gynecology, Helsinki University Hospital, University of Helsinki, Helsinki, Finland; ${ }^{2}$ Unit of Systems Toxicology, Finnish Institute of Occupational Health, Helsinki, Finland; ${ }^{3}$ Department of Oncology, Helsinki University Hospital, University of Helsinki, Helsinki, Finland; ${ }^{4}$ Department of Clinical Genetics, Helsinki University Hospital, University of Helsinki, Helsinki, Finland; ${ }^{5}$ Netherlands Cancer Institute, Antoni van Leeuwenhoek hospital, Amsterdam, The Netherlands; ${ }^{6}$ Centre for Cancer Genetic Epidemiology, Department of Oncology, University of Cambridge, Cambridge, UK; ${ }^{7}$ Centre for Cancer Genetic Epidemiology, Department of Public Health and Primary Care, University of Cambridge, Cambridge, UK; ${ }^{8} \mathrm{Clinical}$ Gerontology, Department of Public Health and Primary Care, University of Cambridge, Cambridge, UK; ${ }^{9}$ Faculty of Health and Medical Sciences, University of Copenhagen, Copenhagen, Denmark; ${ }^{10}$ Copenhagen General Population Study, Herlev Hospital, Copenhagen University Hospital, Herlev, Denmark; ${ }^{11}$ Department of Clinical Biochemistry, Herlev Hospital, Copenhagen University Hospital, Herlev, Denmark; ${ }^{12}$ Division of Genetics and Epidemiology, The Institute of Cancer Research, London, UK; ${ }^{13}$ Division of Breast Cancer Research, The Institute of Cancer Research, London, UK; ${ }^{14}$ Division of Cancer Epidemiology and Genetics, National Cancer Institute, Rockville, Maryland, USA; ${ }^{15}$ Gynaecology Research Unit, Hannover Medical School, Hannover, Germany; ${ }^{16}$ Department of Radiation Oncology, Hannover Medical School, Hannover, Germany; ${ }^{17}$ Department of Medical Epidemiology and Biostatistics, Karolinska Institutet, Stockholm, Sweden; ${ }^{18}$ Department of Genetics and Fundamental Medicine, Bashkir State University, Ufa, Russia; ${ }^{19}$ Institute of Biochemistry and Genetics, Ufa Scientific Center of Russian Academy of Sciences, Ufa, Russia; ${ }^{20}$ Department of Genetics, Institute for Cancer Research, Radiumhospitalet, Oslo University Hospital, University of Oslo, Oslo, Norway; ${ }^{21}$ Department of Clinical Molecular Biology, Oslo University Hospital, Oslo, Norway; ${ }^{22}$ K.G. Jebsen Center for Breast Cancer Research, Institute of Clinical Medicine, Faculty of Medicine, University of Oslo, Oslo, Norway; ${ }^{23}$ Department of Oncology, Radiumhospitalet, Oslo University Hospital, University of Oslo, Oslo, Norway; ${ }^{24}$ Department of Radiology, Radiumhospitalet, Oslo University Hospital, University of Oslo, Oslo, Norway; ${ }^{25}$ National Resource Centre for Long-term Studies after Cancer, Cancer Clinic, Radiumhospitalet, Oslo University Hospital, University of Oslo, Oslo, Norway; ${ }^{26}$ Department of Breast and Endocrine Surgery, Institute for Clinical Medicine, Ullevaal University Hospital, Oslo, Norway; ${ }^{27}$ Department of Clinical Molecular Biology, Institute of Clinical Medicine, Akershus University Hospital, Lørenskog, Norway; ${ }^{28}$ Department of Oncology, Ullevaal University Hospital, University of Oslo, Oslo, Norway; ${ }^{29}$ Department of Pathology, Akershus University Hospital, Lørenskog, Norway; ${ }^{30}$ Department of Surgery, Akershus University Hospital, Lørenskog, Norway; ${ }^{31}$ Department of Oncology, Haukeland University Hospital, Bergen, Norway; ${ }^{32}$ Section of Oncology, Institute of Medicine, University of Bergen, Bergen, Norway; ${ }^{33}$ Norwegian Centre for Integrated Care and Telemedicine, University Hospital of North Norway, Troms $ø$, Norway; ${ }^{34}$ Department of Community Medicine, Faculty of Health Sciences, University of Tromsø-The Arctic University of Norway, Tromsø, Norway; ${ }^{35}$ Department of Non-Communicable Disease Epidemiology, London School of Hygiene and Tropical Medicine, London, UK; ${ }^{36}$ Department of Laboratory Medicine and Pathology, Mayo Clinic, Rochester, Minnesota, USA; ${ }^{37}$ Department of Health Sciences Research, Mayo Clinic, Rochester, Minnesota, USA; ${ }^{38}$ German Cancer Consortium (DKTK), German Cancer Research Center (DKFZ), Heidelberg, Germany; ${ }^{39}$ Dr. Margarete Fischer-Bosch-Institute of Clinical Pharmacology, Stuttgart, Germany; ${ }^{40}$ University of Tübingen, Tübingen, Germany; ${ }^{41}$ Molecular Genetics of Breast Cancer, German Cancer Research Center (DKFZ), Heidelberg, Germany; ${ }^{42}$ Molecular Epidemiology Group, German Cancer Research Center (DKFZ), Heidelberg, Germany; ${ }^{43}$ Department of Obstetrics and Gynecology, University of Heidelberg, Heidelberg, Germany; ${ }^{44}$ National Center for Tumor Diseases, University of Heidelberg, Heidelberg, Germany; ${ }^{45}$ Division of Gynaecology and Obstetrics, Technische Universität München, Munich, Germany; ${ }^{46}$ Center for Molecular 
Medicine Cologne (CMMC), University of Cologne, Cologne, Germany; ${ }^{47}$ Center for Hereditary Breast and Ovarian Cancer, University Hospital of Cologne, Cologne, Germany; ${ }^{48}$ Center for Integrated Oncology (CIO), University Hospital of Cologne, Cologne, Germany; ${ }^{49}$ Sheffield Cancer Research, Department of Oncology, University of Sheffield, Sheffield, UK; ${ }^{50}$ Academic Unit of Pathology, Department of Neuroscience, University of Sheffield, Sheffield, UK; ${ }^{51}$ Research Oncology, Guy's Hospital, King's College London, London, UK; ${ }^{52}$ Wellcome Trust Centre for Human Genetics and Oxford NIHR Biomedical Research Centre, University of Oxford, Oxford, UK; ${ }^{53}$ Laboratory for Translational Genetics, Department of Oncology, University of Leuven, Leuven, Belgium; ${ }^{54}$ Vesalius Research Center, VIB, Leuven, Belgium; ${ }^{55}$ Department of Molecular Medicine and Surgery, Karolinska Institutet, Stockholm, Sweden; ${ }^{56}$ Department of Oncology - Pathology, Karolinska Institutet, Stockholm, Sweden; ${ }^{57}$ Department of Medical Oncology, Family Cancer Clinic, Erasmus MC Cancer Institute, Rotterdam, The Netherlands; ${ }^{58}$ Department of Gynaecology and Obstetrics, University Hospital Erlangen, Friedrich-Alexander University Erlangen-Nuremberg, Comprehensive Cancer Center Erlangen-EMN, Erlangen, Germany; ${ }^{59}$ David Geffen School of Medicine, Department of Medicine, Division of Hematology and Oncology, University of California at Los Angeles, Los Angeles, California, USA; ${ }^{60}$ Department of Molecular Genetics, University of Toronto, Toronto, Ontario, Canada; ${ }^{61}$ Lunenfeld-Tanenbaum Research Institute of Mount Sinai Hospital, Toronto, Ontario, Canada; ${ }^{62}$ Division of Epidemiology, Dalla Lana School of Public Health, University of Toronto, Toronto, Ontario, Canada; ${ }^{63}$ Prosserman Centre for Health Research, Lunenfeld-Tanenbaum Research Institute of Mount Sinai Hospital, Toronto, Ontario, Canada; ${ }^{64}$ Department of Epidemiology, University of California Irvine, Irvine, California, USA; ${ }^{65} \mathrm{Centre}$ for Epidemiology and Biostatistics, Melbourne School of Population and Global health, The University of Melbourne, Melbourne, Australia; ${ }^{66}$ Cancer Epidemiology Centre, Cancer Council Victoria, Melbourne, Australia; ${ }^{67}$ Division of Preventive Oncology, German Cancer Research Center (DKFZ), Heidelberg, Germany; ${ }^{68}$ Division of Clinical Epidemiology and Aging Research, German Cancer Research Center (DKFZ), Heidelberg, Germany; ${ }^{69}$ Institute of Clinical Medicine, Pathology and Forensic Medicine, University of Eastern Finland, Kuopio, Finland; ${ }^{70}$ Cancer Center, Kuopio University Hospital, Kuopio, Finland; ${ }^{71}$ Imaging Center, Department of Clinical Pathology, Kuopio University Hospital, Kuopio, Finland; ${ }^{72}$ Division of Cancer Epidemiology, German Cancer Research Center (DKFZ), Heidelberg, Germany; ${ }^{73}$ Department of Human Genetics, Leiden University Medical Center, Leiden, The Netherlands; ${ }^{74}$ Department of Pathology, Leiden University Medical Center, Leiden, The Netherlands; ${ }^{75}$ Genetic Epidemiology Laboratory, Department of Pathology, The University of Melbourne, Melbourne, Australia; ${ }^{76}$ Department of Epidemiology, Cancer Prevention Institute of California, Fremont, California, USA $;{ }^{77}$ Department of Health Research and Policy - Epidemiology, ${ }^{78}$ Stanford Cancer Institute, Stanford University School of Medicine, Stanford, California, USA; ${ }^{79}$ Department of Electron Microscopy/ Molecular Pathology, The Cyprus Institute of Neurology and Genetics, Nicosia, Cyprus; ${ }^{80}$ Peter MacCallum Cancer Center, The University of Melbourne, Melbourne, Australia. Correspondence: Heli Nevanlinna (heli.nevanlinna@hus.fi)

\section{REFERENCES}

1. CHEK2 Breast Cancer Case-Control Consortium. CHEK2*1100delC and susceptibility to breast cancer: A collaborative analysis involving 10,860 breast cancer cases and 9,065 controls from 10 studies. Am J Hum Genet 2004;74:1175-1182

2. Weischer M, Bojesen SE, Ellervik C, Tybjaerg-Hansen A, Nordestgaard BG CHEK2*1100delC genotyping for clinical assessment of breast cancer risk: meta-analyses of 26,000 patient cases and 27,000 controls. J Clin Oncol 2008;26:542-548.

3. Cybulski C, Wokołorczyk D, Jakubowska A, et al. Risk of breast cancer in women with a CHEK2 mutation with and without a family history of breast cancer. J Clin Oncol 2011;29:3747-3752.
4. Adank MA, Verhoef $S$, Oldenburg RA, et al. Excess breast cancer risk in first degree relatives of CHEK2*1100delC positive familial breast cancer cases. Eur J Cancer 2013;49:1993-1999.

5. Johnson AD, Handsaker RE, Pulit SL, Nizzari MM, O'Donnell CJ, de Bakker PI. SNAP: a web-based tool for identification and annotation of proxy SNPs using HapMap. Bioinformatics 2008;24:2938-2939.

6. Fletcher O, Johnson N, Dos Santos Silva I, et al. Family history, genetic testing, and clinical risk prediction: pooled analysis of CHEK2 1100delC in 1,828 bilateral breast cancers and 7,030 controls. Cancer Epidemiol Biomarkers Prev 2009;18:230-234.

7. Michailidou K, Hall P, Gonzalez-Neira A, et al.; Breast and Ovarian Cancer Susceptibility Collaboration; Hereditary Breast and Ovarian Cancer Research Group Netherlands (HEBON); KConFab Investigators; Australian Ovarian Cancer Study Group; GENICA (Gene Environment Interaction and Breast Cancer in Germany) Network. Large-scale genotyping identifies 41 new loci associated with breast cancer risk. Nat Genet 2013;45:353-61, 361e1.

8. Mavaddat N, Pharoah PD, Michailidou K, et al. Prediction of breast cancer risk based on profiling with common genetic variants. J Natl Cancer Inst 2015;107:djv036.

9. Anderson WF, Rosenberg PS, Prat A, Perou CM, Sherman ME. How many etiological subtypes of breast cancer: two, three, four, or more? J Nat/ Cancer Inst 2014;106:dju165.

10. Weischer M, Nordestgaard BG, Pharoah P, et al. CHEK2*1100delC heterozygosity in women with breast cancer associated with early death, breast cancer-specific death, and increased risk of a second breast cancer. J Clin Oncol 2012;30: 4308-4316.

11. Cox A, Dunning AM, Garcia-Closas M, et al.; Kathleen Cunningham Foundation Consortium for Research into Familial Breast Cancer; Breast Cancer Association Consortium. A common coding variant in CASP8 is associated with breast cancer risk. Nat Genet 2007;39:352-358.

12. Easton DF, Pooley KA, Dunning AM, et al.; SEARCH collaborators; KConFab; AOCS Management Group. Genome-wide association study identifies novel breast cancer susceptibility loci. Nature 2007;447:1087-1093.

13. Schmidt MK, Hogervorst $F$, van Hien $R$, et al. Age- and tumor subtype-specific breast cancer risk estimates for CHEK2*1100delC carriers. J Clin Oncol 2016;34:2750-2760.

14. Benjamini Y, Hochberg Y. Controlling the false discovery rate: A practical and powerful approach to multiple testing. Journal of royal statistical society. Serier $B$ (Methodological) 1995; 57:289-300

15. Demidenko E. Sample size and optimal design for logistic regression with binary interaction. Stat Med 2008;27:36-46.

16. Johnson N, Fletcher O, Naceur-Lombardelli C, dos Santos Silva I, Ashworth A, Peto J. Interaction between CHEK2*1100delC and other low-penetrance breast-cancer susceptibility genes: a familial study. Lancet 2005;366: 1554-1557.

17. Antoniou AC, Pharoah PD, McMullan G, et al. A comprehensive model for familial breast cancer incorporating BRCA1, BRCA2 and other genes. Br J Cancer 2002;86:76-83.

18. Kuchenbaecker KB, Neuhausen SL, Robson M, et al.; Breast Cancer Family Registry; EMBRACE Study; GEMO Study Collaborators; HEBON; KConFab Investigators; CIMBA. Associations of common breast cancer susceptibility alleles with risk of breast cancer subtypes in BRCA1 and BRCA2 mutation carriers. Breast Cancer Res 2014;16:3416.

19. NICE Guidance. National Collaborating Centre for Cancer (UK). 2013. https:// www.nice.org.uk/guidance/cg164/chapter/Terms-used-in-this-guideline. 